\title{
Hypoplasia of Pancreatic Islets in Transgenic Mice Expressing Activin Receptor Mutants
}

\author{
Takashi Yamaoka, ${ }^{\star}$ Chiyoko Idehara, ${ }^{*}$ Makiko Yano,, ${ }^{*}$ Takaya Matsushita, ${ }^{\star}$ Taketo Yamada, Setsuko li, ${ }^{\star}$ Maki Moritani, ${ }^{\star}$ \\ Jun-ich Hata, Hiromu Sugino, ${ }^{\ddagger}$ Sumihare Noji, ${ }^{\S}$ and Mitsuo Itakura* \\ *Otsuka Department of Clinical and Molecular Nutrition, ${ }^{\ddagger}$ Institute for Enzyme Research, and ${ }^{\S}$ Department of Biological Science and

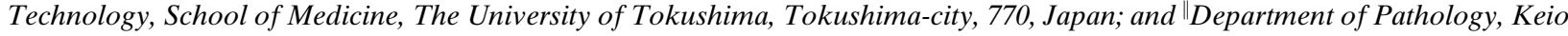 \\ University, School of Medicine, Tokyo, 160-0016, Japan
}

\begin{abstract}
Activin, a member of the TGF- $\beta$ superfamily, regulates the growth and differentiation of a variety of cell types. Based on the expression of activin in pancreatic rudiments of rat embryos and stimulation of insulin secretion from adult rat pancreatic islets by activin, activin is implicated in the development and function of islets. To examine the significance of activin signaling in the fetal and postnatal development of islets, transgenic mice expressing a dominant negative form of activin receptor (dn-ActR) or a constitutively active form of activin receptor (ActR-T206D) in islets were generated together with the transgenic mice expressing intact activin receptor (intact ActR) as a negative control. Transgenic mice with both dn-ActR and ActR-T206D showed lower survival rates, smaller islet area, and lower insulin content in the whole pancreas with impaired glucose tolerance when compared with transgenic mice with intact ActR or littermates, but they showed the same $\alpha$ cell/ $\beta$ cell ratios as their littermates. In addition to islet hypoplasia, the insulin response to glucose was severely impaired in dnActR transgenic mice. It is suggested that a precisely regulated intensity of activin signaling is necessary for the normal development of islets at the stage before differentiation into $\alpha$ and $\beta$ cells, and that activin plays a role in the postnatal functional maturation of islet $\beta$ cells. (J. Clin. Invest. 1998. 102:294-301.) Key words: morphogenesis • cell differentiation - signal transduction • insulin - fetal development
\end{abstract}

\section{Introduction}

Activin is a member of the TGF- $\beta$ superfamily and has diverse actions in a variety of mammalian tissues at many stages during development. Activin stimulates follicle-stimulating hormone secretion in the pituitary gland (1), performs autocrine or paracrine actions in male and female gonads (2), acts in

Address correspondence to Takashi Yamaoka, M.D., Ph.D., Otsuka Department of Clinical and Molecular Nutrition, School of Medicine (Institute for Genome Research), The University of Tokushima, Tokushima-city, 770-8503, Japan. Phone: 81-886-33-7098; FAX: 81886-31-9476; E-mail: yamaoka@nutr.med.tokushima-u.ac.jp

Received for publication 8 January 1998 and accepted in revised form 28 April 1998.

J. Clin. Invest.

(C) The American Society for Clinical Investigation, Inc. 0021-9738/98/07/0294/08 \$2.00

Volume 102, Number 2, July 1998, 294-301

http://www.jci.org erythroid differentiation (3), promotes neuron survival (4), and induces mesoderm in amphibian development $(5,6)$. It was reported recently that activin is expressed in rat islets $(7$, 8) and rat pancreatic anlage (9) on embryonic day 12 (E12), and it stimulates insulin secretion (8) even in the absence of glucose (10) by inhibiting the activity of ATP-sensitive $\mathrm{K}^{+}$ channels and modulating voltage-dependent $\mathrm{Ca}^{2+}$ channels (11). Furthermore, it was reported that betacellulin and activin A coordinately converted the cells of an amylase-secreting pancreatic exocrine rat cell line, AR42J, into insulin-producing cells (12), and activin profoundly altered epithelial branching morphogenesis of embryonic mouse pancreas rudiments in culture (13). In consideration of these reports, we generated transgenic mice with impaired signal transduction of activin in pancreatic endocrine cells to examine whether activin plays a role in the fetal and postnatal development of islets.

For transmembrane signaling, activin requires two types of receptors containing the serine/threonine kinase domain, activin receptor type I (ActR I) and type II (ActR II), as well as the signaling pathways of other members of the TGF- $\beta$ superfamily. Activin binds directly to ActR II and this complex associates with ActR I, resulting in hyperphosphorylation of ActR I by the kinase activity of ActR II (14). Activated serine/ threonine kinase of ActR I phosphorylates Smad proteins, especially Smad2, which are considered to be transcriptional activators of the target genes of activin (15). Truncated ActR II excluding a serine/threonine kinase domain functions as a dominant negative receptor, and blocks activin signaling (16). In contrast, ActR I with the substitution of Thr-206 for Asp (T206D) is constitutively active and can transduce signals independent of ActR II and a ligand (14). In this study, a dominant negative form of ActR II (dn-ActR) or a constitutively active form of ActR I (ActR-T206D) was expressed under the regulation of insulin promoter in islets of transgenic mice.

\section{Methods}

Transgene constructs. For dn-ActR cDNA, the codon of tyrosine, which is the seventh amino acid in the cytoplasmic domain of mouse ActR IIB, was changed to a stop codon (from TAC to TAG) using a PCR-based technique (sense primer; 5'-GGGCCCAGAAGTCACGTACGA-3', and antisense primer; 5'-CTAGGGAGGTTTCCGATGACGAT-3'), and the serine/threonine kinase domain was entirely removed (Fig. 1). The result of mutagenesis was confirmed by

1. Abbreviations used in this paper: ActR, activin receptor; ActRT206D, constitutively active form of the activin receptor; $\mathrm{BDF}_{1}$, C57BL/6 × DBA2; BMP, bone morphogenetic protein; dn-ActR, dominant negative form of the activin receptor; $\mathrm{E}$, embryonic day; $\mathrm{F}_{1}$, $\mathrm{F}_{1}$ of founder mice; PDX-1, pancreatic and duodenal homeobox gene-1; RT, reverse transcriptase. 
the fluorescent automated DNA sequencer (ABI 377; Perkin-Elmer, Division of Applied Biosystems, Foster City, CA). Mouse ActRT206D cDNA was a kind gift from Dr. Huylebroeck (Laboratory of Molecular Biology, University of Leuven, Leuven, Belgium). These two mutants and intact ActR IIB cDNA were cloned into the EcoRI site downstream of the human insulin promoter in the vector containing the exon-intron organization and the polyadenylation signal of rabbit $\beta$-globin gene, respectively (Fig. 1). Transgenic mice expressing an intact form of ActR II were generated as a negative control to rule out the possibility that the overexpression of ActR by itself impairs the development and functions of islets irrespective of activin signaling. An SphI-XhoI fragment of each transgene was purified by agarose gel electrophoresis and the glass powder method with the Gene Clean II Kit (Bio 101 Inc., Vista, CA) and resuspended in 10 $\mathrm{mM}$ Tris- $\mathrm{Cl}(\mathrm{pH} 7.4)$ and $0.1 \mathrm{mM}$ EDTA at the concentration of 500 copies/pl for microinjection.

Generation of transgenic mice. The transgene was microinjected into the male pronuclei of fertilized eggs obtained from superovulated $\mathrm{BDF}_{1}(\mathrm{C} 57 \mathrm{BL} / 6 \times \mathrm{DBA} 2)$ female mice crossed with male $\mathrm{BDF}_{1}$. Injected embryos were implanted in the oviducts of pseudopregnant female mice and allowed to develop (17). DNA was extracted from tail snips of live offspring or from heads of E19.5 embryos by the proteinase K/SDS method (18). The integration of the transgene into the mouse genome was detected by PCR between a sense primer in exon 1 of the human insulin promoter (5'-GCATCAGAAGAGGCCATCAA-3') and an antisense primer in exon 2 of the rabbit $\beta$-globin gene (5'-ACTCACCPCCTGAAGTTCTCAG-3') and Southern blot analysis. An EcoRI fragment of each transgene, i.e., normal or mutated ActR cDNA, was used as a probe. The copy numbers of integrated transgenes were determined from the intensity of each radioactive band in Southern blot analysis compared with indicator bands of 1,10, and 100 copies of the transgene, or the band for the intrinsic ActR gene. 10-40-wk-old mice were used as adult mice in this study.

Expression of intrinsic ActR and transgenes in islets. Islets were obtained from mouse pancreata by the collagenase method (19). Total RNA of islets or cultured cells was extracted with ISOGEN (Nippon Gene, Tokyo, Japan) and reverse transcribed to cDNA with the avian myeloblastosis virus reverse transcriptase (RT) first-strand cDNA

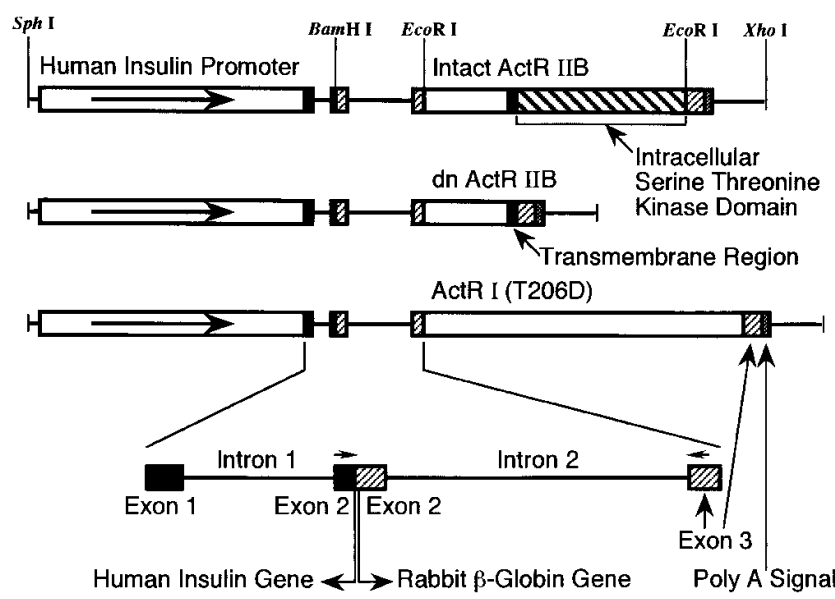

$1.0 \mathrm{~kb}$

Figure 1. Three transgene constructs used in this study. To detect transgene-derived mRNA, two primers encompassing the $\beta$-globin intron 2 including a sense primer in exon 2 of the human insulin promoter (left horizontal arrow) and an antisense primer in exon 3 of the rabbit $\beta$-globin gene (right horizontal arrow) were synthesized for RT-PCR. Although the length of the transgene sequence between these two primers is $\sim 0.6 \mathrm{kbp}$, the size of the RT-PCR product becomes 86 bp because the $\beta$-globin intron 2 is spliced out. synthesis kit (Life Sciences, Inc., St. Petersburg, FL) according to the manufacturer's protocol. Because the expression of ActR I and II in normal mouse islets has not been reported, its presence was ascertained first. ActR I cDNA (sense primer: 5'-CTTGTCTCTGACTATCACGAGCA-3', and antisense primer: 5'-ATATGCAGGTGTGCCAAACCACT-3') and ActR II cDNA (sense primer: 5'-TTTGGCTGCGTTTGGAAGGCTCA-3', and antisense primer: 5'-TTCTCGGCAGCAATGAACTGCAA- $3^{\prime}$ ) were amplified by PCR using the primer pairs shown in parentheses in the samples from normal $\mathrm{BDF}_{1}$ mice and two cell lines of MIN 6 and $\alpha \mathrm{TC}$, which are derived from mouse pancreatic islet $\beta$ and $\alpha$ cells, respectively. PDX-1 (pancreatic and duodenal homeobox gene-1, which was previously referred to as insulin promoter factor-1) cDNA (sense primer: $5^{\prime}$-CGGACATCTCCCCATACGAAGTG-3', and antisense primer: 5'-CGCACAATCTTGCTCCGGCTCTT-3') was also amplified as a positive control. To detect the expression of transgene in transgenic mice, cDNA of the transgene was amplified by PCR between a sense primer (5'-ATCACTGTCCTTCTGCACCTGTCA-3') in exon 2 of the human insulin promoter and an antisense primer $\left(5^{\prime}\right.$-TGAGACAGCACAACAACCAGCA-3') in exon 3 of the rabbit $\beta$-globin gene encompassing its intron 2 (Fig. 1). PCR products were cloned into a pCR II vector (Invitrogen Corp., San Diego, CA) and their identities were confirmed by DNA sequencing.

Histopathological examinations. Fetal mouse bodies and the pancreata of adult mice were fixed with $20 \%$ formalin in PBS, embedded in paraffin, sectioned, and stained with hematoxylin and eosin, aldehyde fuchsin for $\beta$ cells, and Grimelius' stain for $\alpha$ cells. For immunohistochemistry, a guinea pig polyclonal antibody against porcine insulin or glucagon (DAKO JAPAN Co., Ltd., Kyoto, Japan) was used. In a quantitative analysis of islet cell area, the sections of paraffinembedded fetal bodies were immunohistochemically stained at $250-\mu \mathrm{m}$ intervals from diaphragm to ileum, and all slices where pancreas tissue was observed were photographed. The areas of $\alpha$ and $\beta$ cells and of the whole pancreas were determined by tracing the photograph onto graph paper ruled into 1-mm squares, and the area of the whole islets was determined as the sum of both $\alpha$ and $\beta$ cell areas.

Insulin content in the whole pancreas. Each pancreas removed from an adult mouse was homogenized in $4 \mathrm{ml}$ of ice-cold acid-ethanol solution (20), and insulin was extracted at $4^{\circ} \mathrm{C}$ overnight. After centrifugation at $2,000 \mathrm{~g}$ at $4^{\circ} \mathrm{C}$ for $30 \mathrm{~min}$, the supernatant was neutralized and diluted with PBS. Insulin concentrations in the 1,000- or 10,000fold diluted samples were assayed by an ELISA kit for insulin with a mouse insulin standard (Seikagaku Kogyo, Tokyo, Japan).

Glucose tolerance test. After overnight fasting, $1 \mathrm{mg} / \mathrm{g}$ body wt of glucose in physiological saline was intraperitoneally injected. At 0, 30, 60 , and $120 \mathrm{~min}$ after the injection, blood glucose concentrations were determined by the glucose oxidase method with a Diasensor (Kyoto Daiichi Kagaku, Kyoto, Japan). Plasma insulin concentrations were also assayed by the above-mentioned ELISA kit at 0 and $30 \mathrm{~min}$. The insulinogenic index was defined as the ratio of the difference between plasma insulin concentrations (pM) at 0 and $30 \mathrm{~min}$ to the difference between blood glucose concentrations $(\mathrm{mM})$ at 0 and $30 \mathrm{~min}$

Statistical analysis. All data were presented as means \pm SE. For comparison of two means, Student's unpaired $t$ test was used. For comparison of two ratios $\left(Q_{1}, Q_{2}\right)$, a normal distribution curve with a mean of $Q_{1}-Q_{2}$ and a variance of $Q_{1}\left(1-Q_{1}\right) / n_{1}+Q_{2}\left(1-Q_{2}\right) / n_{2}$ was used. $P<0.05$ was considered statistically significant.

\section{Results}

Generation of transgenic mice. To evaluate the influence of transgenes on the survival rate after birth, the generation rate of transgenic mice was examined in E19.5 mouse embryos and 15-wk-old mice that developed from the fertilized eggs microinjected with each transgene. In transgenic mice with intact ActR, the survival rate of 15 -wk-old mice was similar to that of E19.5 embryos (3/32 vs. $2 / 26$, i.e., 8 vs. $9 \%$ ), whereas the sur- 
vival rate of 15 -wk-old mice was significantly lower than that of E19.5 embryos in transgenic mice with dn-ActR (2/30 vs. 6/18, i.e., 7 vs. $33 \%$ ) and with ActR-T206D (3/32 vs. 11/55, i.e., 9 vs. $20 \%$ ). The decreased survival rate after birth as a result of the disturbed activin signaling in pancreatic $\beta$ cells is probably related, at least in part, with insufficient insulin secretion. The surviving adult transgenic mice, two lines with intact ActR, two lines with dn-ActR, and four lines with ActR-T206D, were established as founders and crossed with normal $\mathrm{BDF}_{1}$ to obtain $F_{1}$ mice $\left(F_{1}\right)$. In 15 -wk-old $F_{1}$ of transgenic mice with intact ActR-Tg, the survival rate was 50\% (19/38), thus compatible with Mendel's law, while the survival rates of 15 -wk-old $F_{1}$ with dn-ActR and ActR-T206D were 34\% (25/73) and 31\% (28/91), thus significantly lower than that for $F_{1}$ transgenic mice with intact ActR. Thus, the dn-ActR and ActR-T206D transgenes were shown to exhibit adverse effects on survival rates even in $\mathrm{F}_{1}$ of the surviving adult founders.

Expression of intrinsic ActR and transgenes in islets. By RTPCR with total RNA from islets of $\mathrm{BDF}_{1}$, the expression of intrinsic ActR I and II in islets was ascertained by clear bands of PCR products (data not shown). To further examine the expression of ActR in $\alpha$ and $\beta$ cells, mouse $\alpha$ and $\beta$ cell lines of $\alpha \mathrm{TC}$ and MIN6 were used, respectively. In both cell lines, PCR products from ActR I and II cDNA were clearly amplified (data not shown). The expression of PDX-1, as a control, was detected in mouse islets and MIN6, but not in $\alpha \mathrm{TC}$ (data not shown), while no PCR products were amplified in the samples without RT or a template.

In all lines of transgenic mice with intact ActR, dn-ActR, and ActR-T206D, the expression of each transgene in islets was detected by RT-PCR (Fig. 2), and the amount of PCR products positively correlated with the copy numbers of transgenes with a correlation coefficient of $0.6(n=8)$.

Histopathological examinations. Because transgenic mice with severe islet lesions perish before maturing, E19.5 embryos of transgenic mice were mainly used for the histopathological examination of islets. 3 transgenic embryos with intact ActR, 6 with dn-ActR, and 11 with ActR-T206D were obtained from the fertilized eggs microinjected with the respective transgenes. Islets developed normally in transgenic embryos with intact ActR (Fig. $3 \mathrm{~B}$ ), whereas they developed poorly in dnActR and ActR-T206D transgenic embryos (Fig. 3, $C$ and $D$ ).

Immunohistochemistry of the pancreas in E19.5 embryos against insulin (Fig. 4) and glucagon (data not shown) revealed a decrease in the number of $\beta$ and $\alpha$ cells in $\operatorname{dn}$-ActR and ActR-T206D transgenic mice in parallel with islet hypoplasia. The relative areas of the islets to the whole pancreas in E19.5 transgenic embryos of dn-ActR and ActR-T206D were significantly lower than those in their littermates (Fig. 5). However, the relative areas of $\alpha$ cells to $\beta$ cells in E19.5 embryos are similar in all transgenic mice and littermates and are as follows: $30 \pm 4 \%$ in littermates $(n=6), 29 \pm 4 \%$ in transgenic mice with intact ActR $(n=3), 30 \pm 8 \%$ in dn-ActR transgenic mice $(n=$ $6)$, and $28 \pm 5 \%$ in ActR-T206D transgenic mice $(n=7)$.

In $\mathrm{dn}$-ActR transgenic mice, the relative area of islets to the whole pancreas negatively correlated with the copy number of dn-ActR transgene with a high correlation coefficient of 0.9 (data not shown), i.e., dn-ActR transgenic mice with higher copy numbers of the transgene had more hypoplastic islets than those with lower copy numbers. As expected, the copy numbers of the two surviving founders (4 and 4) were smaller than the average of those of transgenic embryos at E19.5

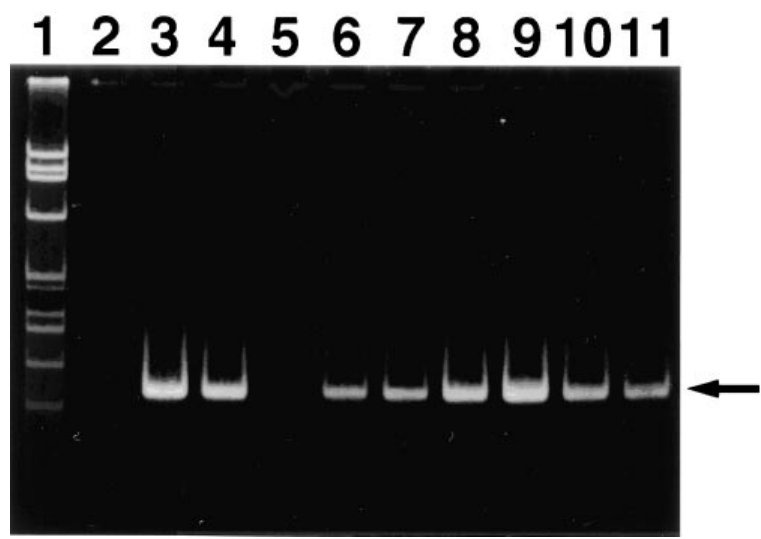

Figure 2. The expression of transgenes in pancreatic islets. Total RNA was extracted from mouse islets collected by the collagenase method and applied to RT-PCR. In all lines of transgenic mice, RTPCR products of 86 bp (arrow) were amplified. Lane 1, Molecular weight standards; lane 2, littermate; lanes 3 and 4, two lines of transgenic mice with intact ActR with copy numbers of 10 and 2, respectively; lane 5, littermate; lanes 6 and 7, two lines of dn-ActR transgenic mice with the same copy number of 4; lanes $8-11$, four lines of ActR-T206D transgenic mice with the copy numbers of 20, 10, 10, and 5, respectively. The amount of PCR products quantified by the intensity of the respective DNA bands positively correlated with the copy numbers of the transgenes with a correlation coefficient of 0.6 $(n=8)$.

$(8.0 \pm 1.2)$. In spite of the low copy numbers, the two surviving founders also showed islet hypoplasia (Fig. 6). Unlike in dnActR transgenic mice, no relationship between the copy number of the transgene and the extent of islet hypoplasia was found in ActR-T206D transgenic mice.

Insulin content in the whole pancreas. Insulin contents $(\mu \mathrm{g} /$ pancreas) were as follows: $13.8 \pm 1.6(n=10$, littermates $)$, 14.0 $\pm 0.7(n=10$, transgenic mice with intact ActR), 6.2 \pm 0.7 $(n=10$, dn-ActR), and 10.1 $\pm 1.0(n=9$, ActR-T206D). Transgenic mice with dn-ActR and ActR-T206D had significantly smaller amounts of insulin in their pancreas than their littermates and transgenic mice with intact ActR. The histogram (data not shown) of these four values was very similar to that in Fig. 5. The islet size and insulin content in dn-ActR transgenic mice seemed to be smaller than those in ActR-T206D transgenic mice, although there was no statistically significant difference between these two types expressing ActR mutants.

Glucose tolerance test. Transgenic mice with intact ActR showed normal glucose tolerance. Because islet hypoplasia in adult dn-ActR and adult ActR-T206D transgenic mice was relatively mild compared with the lesions observed in E19.5 transgenic embryos, fasting blood glucose levels in all transgenic mice and littermates were within the normal range $(<5.5 \mathrm{mM})$. However, dn-ActR and ActR-T206D transgenic mice had significantly higher glycemia both before and after intraperitoneal glucose injection than their littermates (Fig. 7).

To evaluate insulin secretion in transgenic mice, the increase in plasma insulin concentration was determined 30 min after glucose loading (Fig. 8). The responses of serum insulin to glucose administration in intact ActR transgenic mice and littermates were comparable. In ActR-T206D transgenic mice, the increase in serum insulin concentration was smaller than that in the littermates, but the rates of insulin concentration in- 


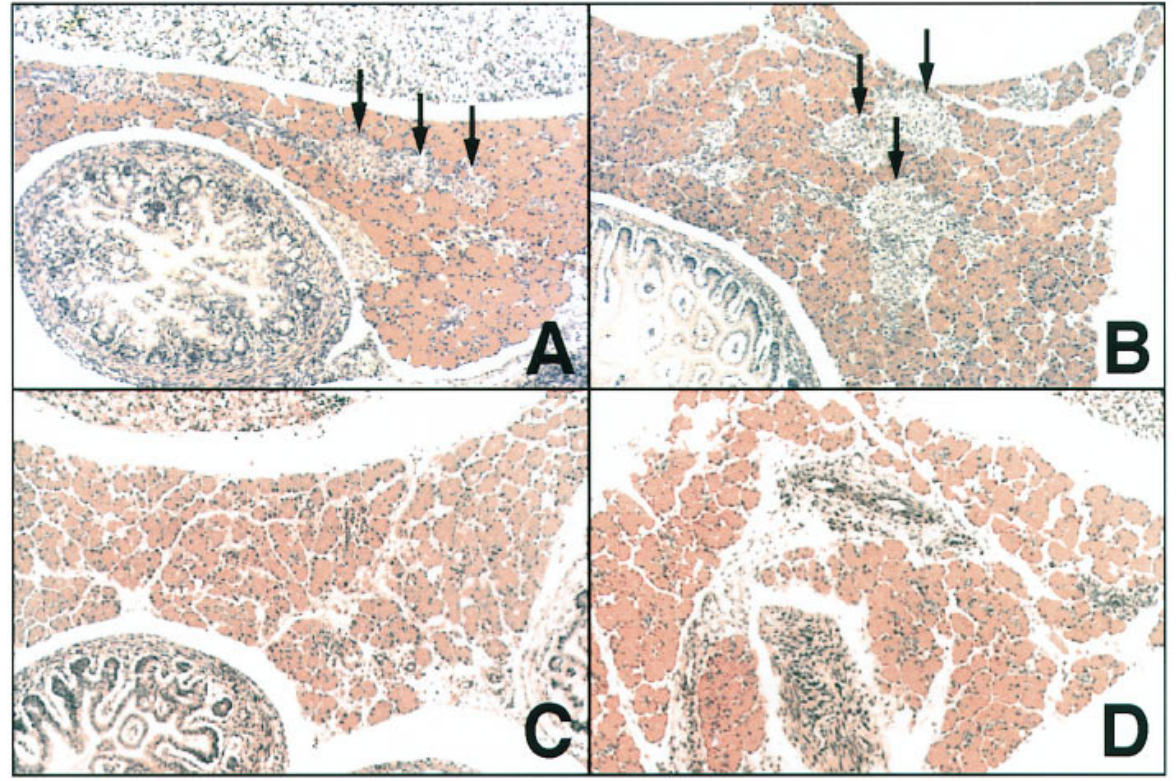

Figure 3. Hematoxylin and eosin staining of the pancreas in E19.5 embryos. $(A)$ Littermate; $(B)$ transgenic mouse with intact ActR; $(C)$ dn-ActR transgenic mouse; and (D) ActR-T206D transgenic mouse. Several normally developed islets are indicated by arrows in $A$ and $B$, but almost no obvious islets were observed in $C$ and $D$. creases were comparable, i.e., 4.5-fold for ActR-T206D transgenic mice and 4.6-fold for the littermates. In contrast, serum insulin concentration in dn-ActR transgenic mice increased poorly in response to glucose by only a factor of 1.5 . Because of the small increase in serum insulin, the insulinogenic index $(\Delta \mathrm{IRI} 30 \mathrm{~min} / \Delta \mathrm{BG} 30 \mathrm{~min})$ in $\mathrm{dn}$-ActR transgenic mice showed a much lower value $(9 \pm 5, n=10)$ than in the littermates $(92 \pm 18, n=20)$ or in transgenic mice with intact ActR $(94 \pm 14, n=10)$ or ActR-T206D $(57 \pm 11, n=10)$.

\section{Discussion}

Generation of transgenic mice. From the generation rates in E19.5 transgenic embryos and 15-wk-old transgenic mice, which developed from fertilized eggs microinjected with the respective transgenes, and in $F_{1}$ mice of the founders, it was demonstrated that the expression of ActR mutants in pancreatic $\beta$ cells results in a decrease in the survival rate of transgenic mice. Although murine pups with complete insulin deficiency were reported to die within a few days after birth (21, 22), our transgenic mice expressing ActR mutants seemed to survive longer. In fact, several founders with ActR mutants died in young adulthood before the age of $15 \mathrm{wk}$.

Expression of intrinsic ActR and transgenes in islets. Although activin has been reported to be expressed in islets $(7,8)$ and pancreatic anlage (9), the expression of ActR I and II in islets was not known at the beginning of this study. ActR I was expressed together with ActR II both in $\alpha \mathrm{TC}$ and MIN6, in addition to islets. These results suggest that ActR I and II are expressed in both $\alpha$ and $\beta$ cells of mouse islets in vivo. The expression of PDX-1 in MIN6, but not in $\alpha$ TC, showed that these

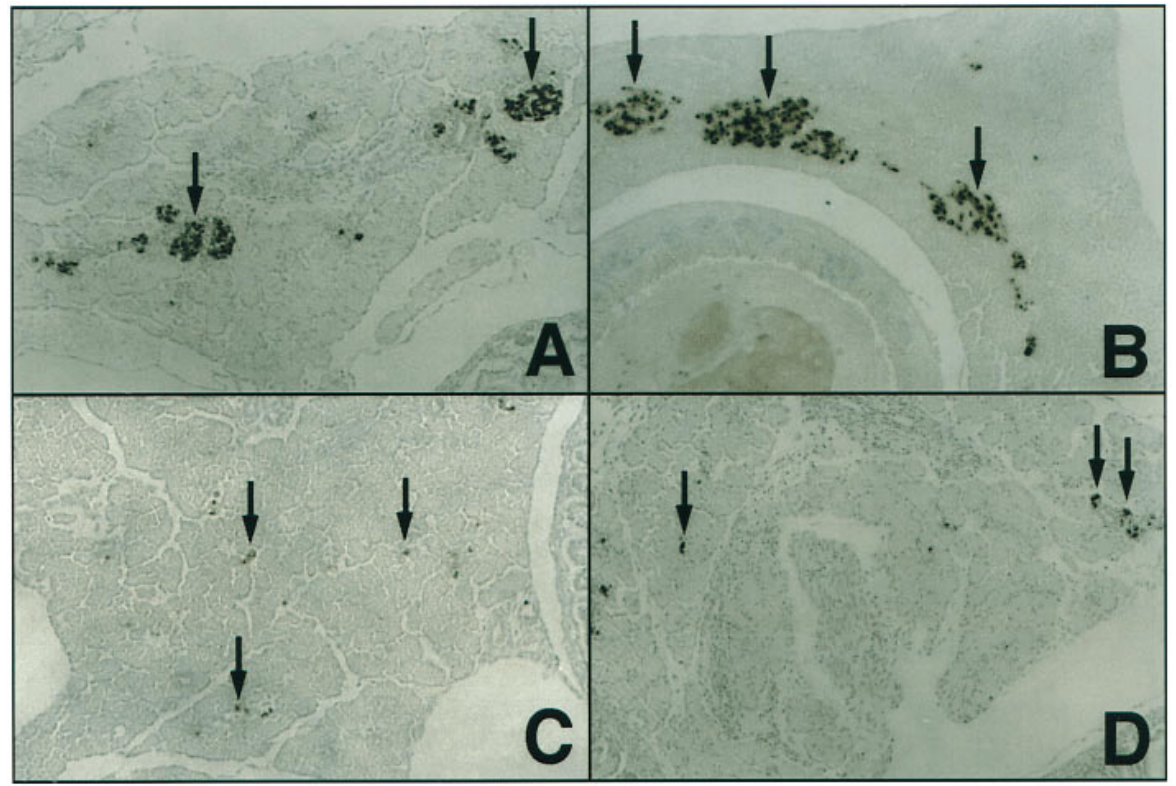

Figure 4. Antiinsulin immunohistochemistry of the pancreas in E19.5 embryos. $(A)$ Littermate; $(B)$ transgenic mouse with intact ActR; $(C)$ dn-ActR transgenic mouse; (D) ActR-T206D transgenic mouse. Insulin-producing cells stained dark brown ( $a r-$ rows) are observed in $A$ and $B$, but are decreased in number in $C$ and $D$. 


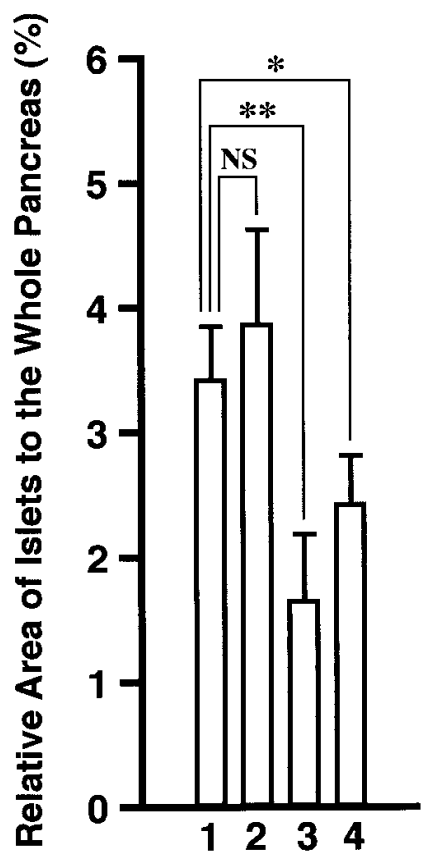

Figure 5. Relative area of islets to the whole pancreas. (1) Littermates $(n=6)$; (2) transgenic mice with intact ActR $(n=3)$; (3) dn-ActR transgenic mice $(n=6)$; and (4) ActR-T206D transgenic mice $(n=7) . * P<0.05$ and $* * P<0.01$.

two culture cell lines retain, at least in part, their original characteristics.

The expression of transgene was detected in all lines of transgenic mice by RT-PCR (Fig. 2). The copy number of transgenes integrated into the mouse genome examined by Southern blot analysis correlated with the amount of PCR product, which suggests that the copy number of the transgene is an indicator of the expression level. In general, the expression level of the transgene depends on the position in the genome, where the transgene was integrated, through the heterochromatin effect. However, if a locus control region, which is responsible for initiating and maintaining a stable and tissuespecific open chromatin structure of a locus, is included in a

transgene, the level of transgene expression becomes independent of position in the genome in proportion to its copy number $(23,24)$. Because the insulin promoter is known to be one of the most powerful tissue-specific promoters when it is used for transgenic mice, and because the transgenes were expressed under the insulin promoter in a copy number-dependent manner in this study, a local control region may be included in the human insulin promoter up to $1.9 \mathrm{~kb}$ upstream of the start codon, ATG (Fig. 1).

Histopathological examinations and insulin content. Islet hypoplasia was observed both in dn-ActR and ActR-T206D transgenic mice (Figs. 3 and 4). This indicates that activin or activin-like signaling at an appropriate intensity is necessary for the development of normal islets. In contrast to transgenic mice expressing the mutated ActR, islets developed normally in intact ActR transgenic mice, which suggests that the number of ActR copies is not a limiting factor for activin signaling in islets. It is well-known that activin can induce embryonic Xenopus cells to develop into several different cell types in a concentration-dependent manner (25-27). Similarly, our data suggest that the intensity of activin or activin-like signaling is an important factor for normal development of islets.

Although both dn-ActR and ActR-T206D transgenic mice showed hypoplastic islets, dn-ActR transgenic mice seemed to have more severe lesions than those with ActR-T206D, according to the data for the relative area of islets (Fig. 5) and insulin content in the whole pancreas in both types of transgenic mice. Moreover, the copy number of the transgene correlated with islet hypoplasia only in dn-ActR, but not in ActR-T206D transgenic mice. These results imply that activin signaling is disturbed even by a modest overexpression of constitutively active ActR-T206D, and that the extent of disturbance easily reaches the plateau level. In contrast, as the amount of dnActR in islets increases, the activin signaling may be blocked to a proportionally greater extent, resulting in gene dosedependent hypoplastic islets in dn-ActR transgenic mice.

In spite of the use of the insulin promoter, the development of $\alpha$ and $\beta$ cells was simultaneously impaired in $\operatorname{dn}-A c t R$ and ActR-T206D transgenic mice, and the ratio of $\alpha$ cells to $\beta$ cells

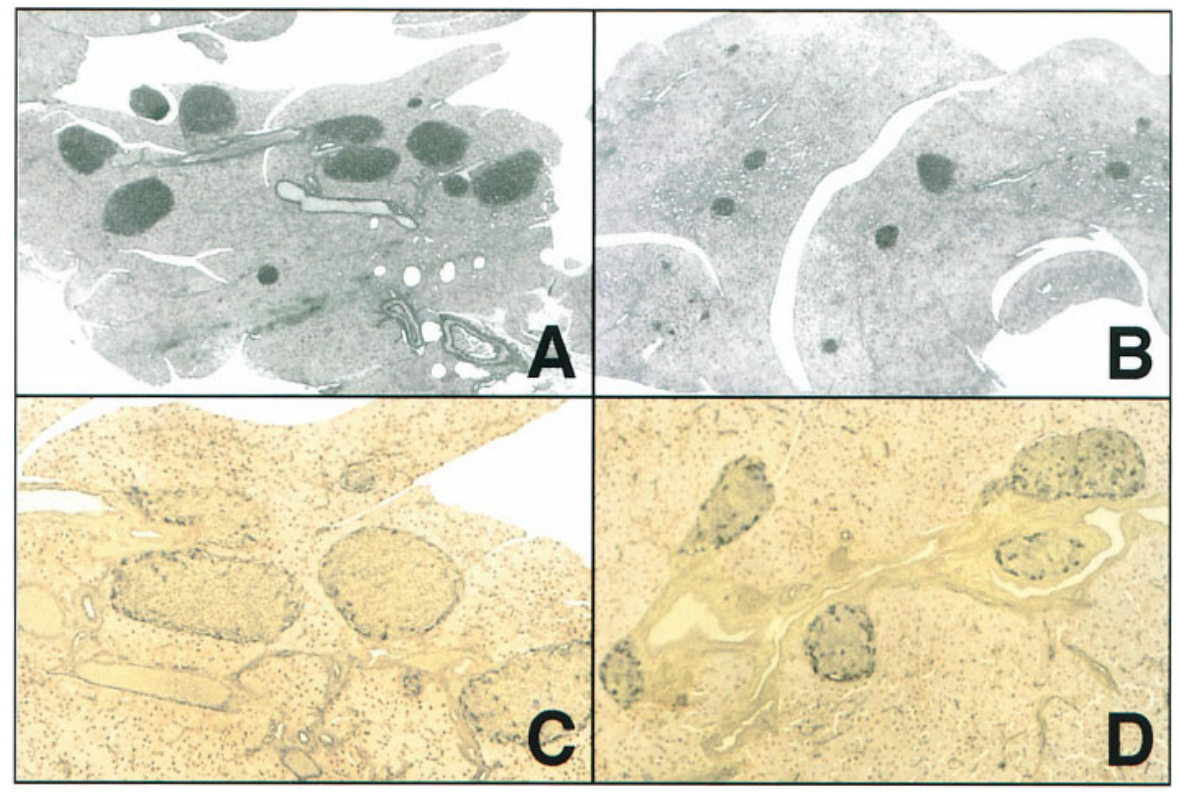

Figure 6. Aldehyde fuchsin staining and Grimelius' staining of the pancreas in 20wk-old mice. Islet $\beta$ cells are stained dark violet with aldehyde fuchsin ( $A$ and $B$ ), and $\alpha$ cells are stained black with Grimelius' stain $(C$ and $D) . A$ and $B$, or $C$ and $D$, are identical magnifications. $A$ and $C$ are littermates; $B$ and $D$ are dn-ActR transgenic mice. Islets in dn-ActR transgenic mice were smaller than those in littermates. 


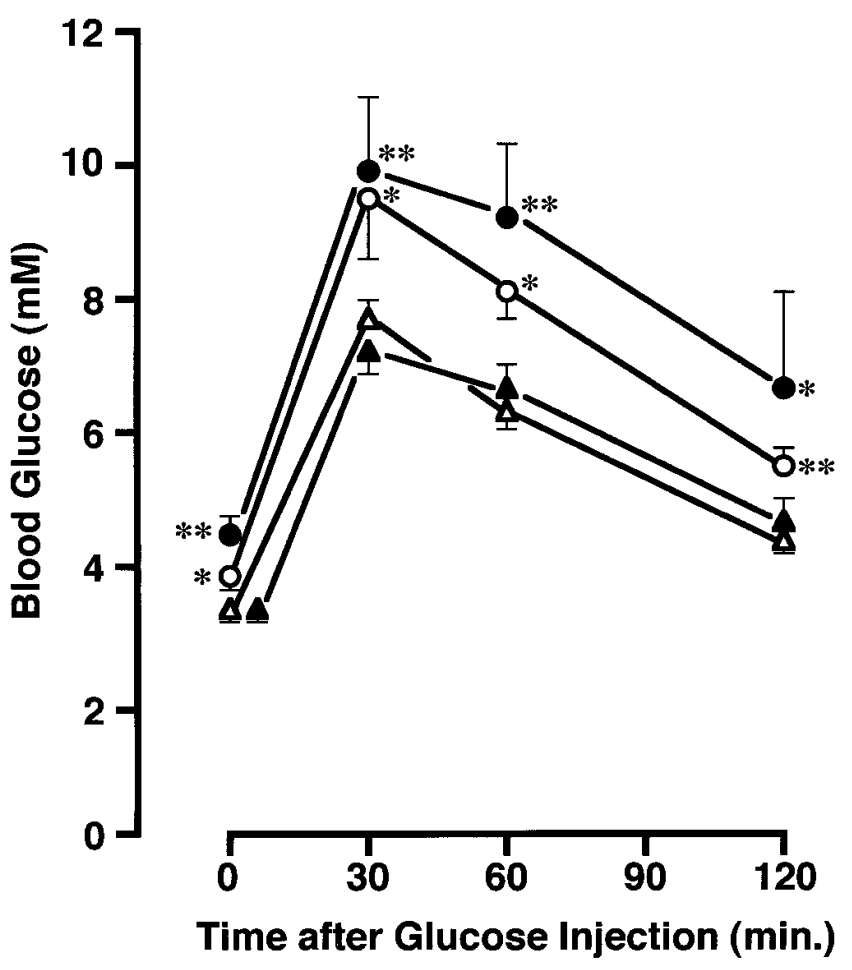

Figure 7. Glucose tolerance test. After $16 \mathrm{~h}$ of fasting, $1 \mathrm{mg} / \mathrm{g}$ body wt of glucose in physiological saline was intraperitoneally injected. Open triangles, littermates $(n=27)$; filled triangles, transgenic mice with intact ActR ( $n=10 ; 5$ of line 1 and 5 of line 2); open circles, dn-ActR transgenic mice ( $n=11 ; 5$ of line 1 and 6 of line 2); filled circles, ActR-T206D transgenic mice $(n=10 ; 3$ of line 1, 1 of line 2, 3 of line 3 , and 3 of line 4). Single and double asterisks indicate statistical significance compared with the values of littermates for $P<0.05$ and 0.01 , respectively.

in both types was similar to the values in littermates and transgenic mice with intact ActR. It is unlikely that the dn-ActR or ActR-T206D was expressed in $\alpha$ cells of these transgenic mice to the extent of disturbing $\alpha$ cell development, because $1.9 \mathrm{~kb}$ of the human insulin promoter used in this study is sufficient for $\beta$ cell-specific expression in transgenic mice (28). It is also unlikely that interactions between $\alpha$ and $\beta$ cells are necessary for the normal $\alpha$ cell development, because $\alpha$ cells normally developed and aggregated into islet-like clusters devoid of $\beta$ cells in transgenic mice expressing dominant negative mutant of mouse cadherin in $\beta$ cells (29). Although the pancreatic islet cell lineage is not fully understood $(30,31)$, coexpression of insulin and glucagon has been observed in the early endocrine cells (32). If insulin/glucagon coexpressing cells are the common precursor cells of $\alpha$ and $\beta$ cells, the growth, differentiation, or survival of these precursor cells may be impaired in dnActR and ActR-T206D transgenic mice. The appearance of the insulin/glucagon coexpressing cells may indicate that promoter function does not become fully restricted to one islet cell type until islets have matured. dn-ActR and ActR-T206D may affect endocrine precursor cells by decreasing the rate of replication or by increasing the rate of apoptosis. Thus, activin or activin-like signaling may play an important role at the early stage of islet cell development before differentiation into $\alpha$ and $\beta$ cells.

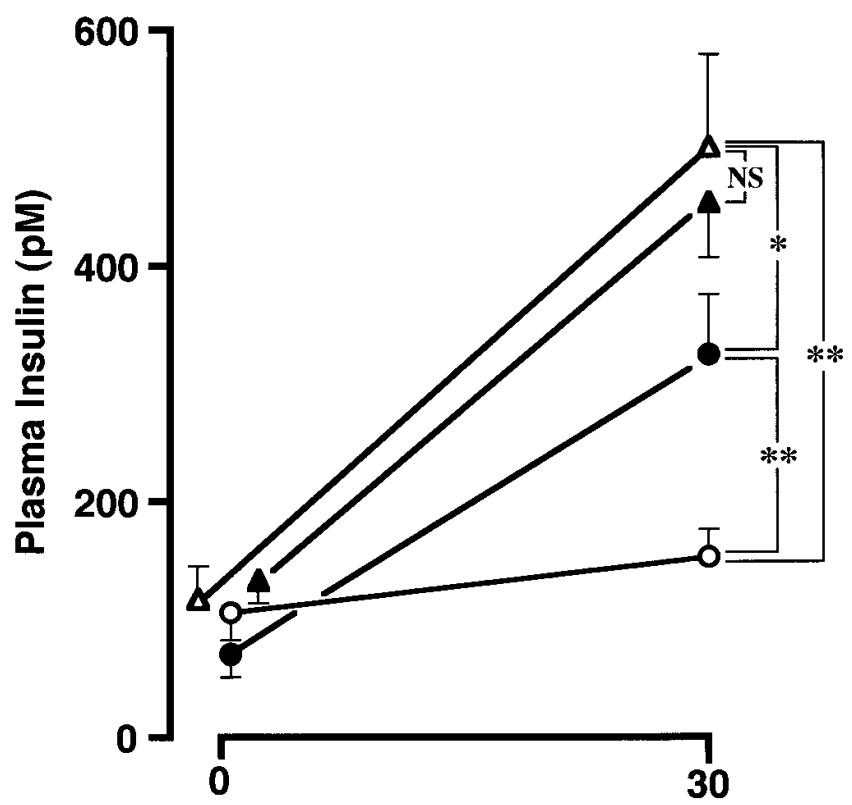

Time after Glucose Injection (min.)

Figure 8. Insulin secretion in response to glucose. Glucose was administered in the same way as in the glucose tolerance test. Open triangles, littermates $(n=20)$; filled triangles, transgenic mice with intact ActR ( $n=10 ; 5$ of line 1 and 5 of line 2); open circles, dn-ActR transgenic mice ( $n=10 ; 5$ of line 1 and 5 of line 2); filled circles, ActR-T206D transgenic mice $(n=10 ; 3$ of line 1,1 of line 2, 3 of line 3 , and 3 of line 4$)$. $* P<0.05$; and $* * P<0.01$.

Glucose tolerance test. Both dn-ActR and ActR-T206D transgenic mice showed impaired glucose tolerance compared with transgenic mice with intact ActR and littermates (Fig. 7). In ActR-T206D animals, the function of islet $\beta$ cells was considered almost normal because the response of insulin secretion to glucose injection was comparable to that in the littermates (a 4.5-fold vs. a 4.6-fold increase, Fig. 8). Therefore, impaired glucose tolerance in ActR-T206D transgenic mice is probably caused by the decrease in islet mass. Because islet $\beta$ cells in these mice responded to glucose to a degree compatible with those of intact ActR transgenic mice and littermates, the maturation of $\beta$ cells seemed to occur so long as activin signaling was not blocked, although an appropriate intensity of activin signaling was necessary for attaining normal islet sizes.

However, in dn-ActR transgenic mice the extremely low response of insulin secretion to glucose (a 1.5-fold increase) cannot be explained only by islet hypoplasia, and suggests rather that decreased activin signaling leads to the dysfunction of $\beta$ cells. The defect of $\beta$ cells in $\mathrm{dn}$-ActR transgenic mice seems to be either in sensing the elevation of blood glucose or in the maximal insulin secretion. The glucose sensitivity of $\beta$ cells has been reported to be acquired after birth, and the response to glucose matures after weaning $(33,34)$. Unlike in adult $\beta$ cells, insulin secretion is not stimulated by glucose in fetal $\beta$ cells, although fetal $\beta$ cells respond to other insulin secretagogues. Activin signaling may be needed for this postnatal maturation of $\beta$ cells. In fact, it was reported that activin at a concentration of $1 \mathrm{nM}$ greatly enhances the insulin response of rat islets to glucose $(8,35)$ and stimulates a signifi- 
cant release of insulin even in glucose-free medium (10) by elevating the cytoplasmic free $\mathrm{Ca}^{2+}$ concentration $(11,36)$.

Redundancy of the TGF- $\beta$ superfamily. Although islet hypoplasia was observed both in dn-ActR and ActR-T206D transgenic mice, activin itself may be dispensable for the normal development of islets. dn-ActR blocks the signaling not only of activin, but also, to a variable degree, of several other members of the TGF- $\beta$ superfamily, including bone morphogenetic protein (BMP)-2 (37), BMP-4 (37, 38), BMP-7 (osteogenic protein-1) (39), and Vg-1 (40). ActR I can be associated with BMP receptor II (41) which binds BMP-2, BMP-4, and BMP-7, and ActR II can also be associated with BMP receptor I (39). In regard to the ligand-receptor interaction and the association between receptors I and II, much redundancy is observed between ActR and BMP receptors. On the other hand, the activin-induced phosphorylation pattern of Smad, the signal transduction molecule of the TGF- $\beta$ superfamily, is very similar to that by TGF- $\beta$. Both activin and TGF- $\beta$ induce phosphorylation of Smad2 (42) and Smad3 (43). In the case where mRNA encoding TGF- $\beta$ receptors is introduced into Xenopus embryos, TGF- $\beta$, like activin, can induce mesoderm (44), although TGF- $\beta$ has no effect on wild Xenopus embryos. Indeed, TGF- $\beta$ promotes the development of endocrine cells and inhibits the development of acinar tissue in three-dimensional gel culture of mouse pancreatic rudiments (45), and the expression of a dominant-negative mutant TGF- $\beta$ receptor in transgenic mice by a metallothionein promoter revealed that TGF- $\beta$ negatively controls the growth of acinar cells and is essential for the maintenance of a differentiated acinar phenotype (46), although the development of the pancreas is normal in TGF- $\beta$-deficient mice (47).

Recently, Pax- 6 was shown to be essential for the differentiation of islet $\alpha$ cells in the pancreas using Pax-6 null mice (48), and activin was reported to inhibit Pax-6 expression in the developing spinal cord and to control the differentiation of neurons (49). It is not clear whether activin plays a role in the differentiation of islet cells through the regulation of Pax-6 expression. Because insulin promoter was used for the expression of transgenes in this study, it is impossible in our transgenic mice to elucidate the action of activin on the undifferentiated endocrine cells before insulin promoter begins to work at E9.5 $(30,31)$. This could be circumvented by using PDX-1 promoter (21) or ISL-1 promoter (50), because these promoters start to function from E8.5 (31) in pancreatic buds and E9.0 (50) in all islet cells, respectively. A study of transgenic mice expressing ActR mutants driven by PDX-1 promoter is underway in our laboratory.

In conclusion, islet hypoplasia with a decrease in the number of both $\alpha$ and $\beta$ cells, which is observed in transgenic mice expressing ActR mutants, suggests that activin or activin-like signaling plays an important role in the development of islets in the early stage of islet development before differentiation into $\alpha$ and $\beta$ cells. Based on the impaired insulin response to glucose observed in dn-ActR transgenic mice, it is also concluded that activin plays a role in the postnatal functional maturation of islet $\beta$ cells.

\section{Acknowledgments}

We wish to sincerely thank Dr. Kenichi Yamamura and Dr. Misao Suzuki (Kumamoto University, School of Medicine) for their advice on the transgenic technique.
This study was supported in part by a grant from Otsuka Pharmaceutical Factory Inc. to the Otsuka Department of Clinical and Molecular Nutrition, School of Medicine, The University of Tokushima.

\section{References}

1. Ling, N., S.Y. Ying, N. Ueno, S. Shimasaki, F. Esch, M. Hotta, and R. Guillemin. 1986. Pituitary FSH is released by a heterodimer of the beta-subunits from the two forms of inhibin. Nature. 321:779-782.

2. Halvorson, L.M., and A.H. DeCherney. 1996. Inhibin, activin, and follistatin in reproductive medicine. Fertil. Steril. 65:459-469.

3. Yu, J., L.E. Shao, V. Lemas, A.L. Yu, J. Vaughan, J. Rivier, and W.W. Vale. 1987. Importance of FSH-releasing protein and inhibin in erythrodifferentiation. Nature. 330:765-767.

4. Schubert, D., H. Kimura, M. LaCorbiere, J. Vaughan, D. Karr, and W.H. Fischer. 1990. Activin is a nerve cell survival molecule. Nature. 344:868-870.

5. Smith, J.C., B.M. Price, K. Van Nimmen, and D. Huylebroeck. 1990. Identification of a potent Xenopus mesoderm-inducing factor as a homologue of activin A. Nature. 345:729-731.

6. Thomsen, G., T. Woolf, M. Whitman, S. Sokol, J. Vaughan, W.W. Vale, and D.A. Melton. 1990. Activins are expressed early in Xenopus embryogenesis and can induce axial mesoderm and anterior structures. Cell. 63:485-493.

7. Ogawa, K., K. Abe, N. Kurosawa, M. Kurohmaru, H. Sugino, M. Takahashi, and Y. Hayashi. 1993. Expression of alpha, beta A and beta B subunits of inhibin or activin and follistatin in rat pancreatic islets. FEBS Lett. 319:217-220.

8. Yasuda, H., K. Inoue, H. Shibata, T. Takeuchi, Y. Eto, Y. Hasegawa, N. Sekine, Y. Totsuka, T. Mine, and E. Ogata. 1993. Existence of activin-A in A- and D-cells of rat pancreatic islets. Endocrinology. 133:624-630.

9. Furukawa, M., Y. Eto, and I. Kojima. 1995. Expression of immunoreactive activin A in fetal rat pancreas. Endocrinol. J. 42:63-68.

10. Furukawa, M., R. Nobusawa, H. Shibata, Y. Eto, and I. Kojima. 1995. Initiation of insulin secretion in glucose-free medium by activin A. Mol. Cell. Endocrinol. 113:83-87.

11. Mogami, H., M. Kanzaki, R. Nobusawa, Y.Q. Zhang, M. Furukawa, and I. Kojima. 1995. Modulation of adenosine triphosphate-sensitive potassium channel and voltage-dependent calcium channel by activin A in HIT-T15 cells. Endocrinology. 136:2960-2966.

12. Mashima, H., H. Ohnishi, K. Wakabayashi, T. Mine, J. Miyagawa, T. Hanafusa, M. Seno, H. Yamada, and I. Kojima. 1996. Betacellulin and activin A coordinately convert amylase-secreting pancreatic AR42J cells into insulinsecreting cells. J. Clin. Invest. 97:1647-1654.

13. Ritvos, O., T. Tuuri, M. Eramaa, K. Saino, K. Hilden, L. Saxen, and S.F. Gilbert. 1995. Activin disrupts epithelial branching morphogenesis in developing glandular organs of the mouse. Mech. Dev. 50:229-245.

14. Attisano, L., J.L. Wrana, E. Montalvo, and J. Massague. 1996. Activation of signaling by the activin receptor complex. Mol. Cell. Biol. 16:1066-1073.

15. Lagna, G., A. Hata, A. Hemmati-Brivanlou, and J. Massague. 1996. Partnership between DPC4 and SMAD proteins in TGF- $\beta$ signaling pathways. Nature. 383:832-836.

16. Hemmati-Brivanlou, A., and D.A. Melton. 1992. A truncated activin receptor inhibits mesoderm induction and formation of axial structures in Xenopus embryos. Nature. 359:609-614.

17. Yamaoka, T., C. Nishimura, K. Yamashita, M. Itakura, T. Yamada, J. Fujimoto, and K. Kokai. 1995. Acute onset of diabetic pathological changes in transgenic mice with human aldose reductase cDNA. Diabetologia. 38:255-261.

18. Hogan, B., R. Beddington, F. Costantini, and E. Lacy. 1994. Manipulating the Mouse Embryo. A Laboratory Manual. 2nd ed. Cold Spring Harbor Laboratory Press, New York.

19. Moritani, M., K. Yoshimoto, S. Ii, M. Kondo, H. Iwahana, T. Yamaoka, T. Sano, N. Nakano, H. Kikutani, and M. Itakura. 1996. Prevention of adoptively transferred diabetes in nonobese diabetic mice with IL-10-transduced islet-specific Th1 lymphocytes. J. Clin. Invest. 98:1851-1859.

20. Yamada, T., J. Brunstedt, and T. Solomon. 1983. Chronic effects of caerulein and secretin on the endocrine pancreas of the rat. Am. J. Physiol. 244: G541-G545.

21. Jonsson, J., L. Carlsson, T. Edlund, and H. Edlund. 1994. Insulin-promoter-factor 1 is required for pancreas development in mice. Nature. 371:606609

22. Sosa-Pineda, B., K. Chowdhury, M. Torres, G. Oliver, and P. Gruss. 1997. The Pax 4 gene is essential for differentiation of insulin-producing $\beta$ cells in the mammalian pancreas. Nature. 386:399-402.

23. Festenstein, R., M. Tolaini, P. Corbella, C. Mamalaki, J. Parrington, M. Fox, A. Miliou, M. Jones, and D. Kiousis. 1996. Locus control region function and heterochromatin-induced position effect variegation. Science. 271:11231125 .

24. Milot, E., J. Strouboulis, T. Trimborn, M. Wijgerde, E. de Boer, A. Langeveld, K. Tan-Un, W. Vergeer, N. Yannoutsos, F. Grosveld, and P. Fraser. 1996. Heterochromatin effects on the frequency and duration of LCR-mediated gene transcription. Cell. 87:105-114.

25. Green, J.B., H.V. New, and J.C. Smith. 1992. Responses of embryonic 
Xenopus cells to activin and FGF are separated by multiple dose thresholds and correspond to distinct axes of the mesoderm. Cell. 71:731-739.

26. Gurdon, J.B., P. Harger, A. Mitchell, and P. Lemaire. 1994. Activin signaling and response to a morphogen gradient. Nature. 371:487-492.

27. Reilly, K.M., and D.A. Melton. 1996. Short-range signaling by candidate morphogens of the TGF- $\beta$ family and evidence for a relay mechanism of induction. Cell. 86:743-754.

28. Goodman, P.A., O. Medina-Martinez, and C. Fernandez-Mejia. 1996. Identification of the human insulin negative regulatory element as a negative glucocorticoid response element. Mol. Cell. Endocrinol. 120:139-146.

29. Dahl, U., A. Sjoedin, and H. Semb. 1996. Cadherins regulate aggregation of pancreatic $\beta$-cells in vivo. Development. 122:2895-2902.

30. Slack, J.M.W. 1995. Developmental biology of the pancreas. Development. 121:1569-1580.

31. Sander, M., and M.S. German. 1997. The $\beta$ cell transcription factors and development of the pancreas. J. Mol. Med. 75:327-340.

32. Alpert, S., D. Hanahan, and G. Tertelman. 1988. Hybrid insulin genes reveal a developmental lineage for pancreatic endocrine cells and imply a relationship with neurons. Cell. 53:295-308.

33. Otonkoski, T., S. Andersson, M. Knip, and O. Simell. 1988. Maturation of insulin response to glucose during human fetal and neonatal development. Diabetes. 37:286-291.

34. Weinhaus, A., P. Poronnik, D. Cook, and B. Tuch. 1995. Insulin secretagogues, but not glucose, stimulate an increase in $\left[\mathrm{Ca}^{2+}\right]_{\mathrm{i}}$ in the fetal rat beta-cell. Diabetes. 44:118-124.

35. Totsuka, Y., M. Tabuchi, I. Kojima, H. Shibai, and E. Ogata. 1988. A novel action of activin A: stimulation of insulin secretion in rat pancreatic islets. Biochem. Biophys. Res. Commun. 156:335-359.

36. Shibata, H., H. Yasuda, N. Sekine, T. Mine, Y. Totsuka, and I. Kojima. 1993. Activin A increases intracellular free calcium concentrations in rat pancreatic islets. FEBS Lett. 329:194-198.

37. Hemmati-Brivanlou, A., and G.H. Thomsen. 1995. Ventral mesodermal patterning in Xenopus embryos: expression patterns and activities of BMP-2 and BMP-4. Dev. Genet. 17:78-89.

38. Chang, C., P.A. Wilson, L.S. Mathews, and A. Hemmati-Brivanlou. 1997. A Xenopus type I activin receptor mediates mesodermal but not neural specification during embryogenesis. Development. 124:827-837.

39. Yamashita, H., P. ten Dijke, D. Huylebroeck, T.K. Sampath, M. Andries, J.C. Smith, C.H. Heldin, and K. Miyazono. 1995. Osteogenic protein-1 binds to activin type II receptors and induces certain activin-like effects. J. Cell Biol. 130:217-226.

40. Schulte-Merker, S., J.C. Smith, and L. Dale. 1994. Effects of truncated activin and FGF receptors and of follistatin on the inducing activities of $\mathrm{Vg} 1$ and activin: does activin play a role in mesoderm induction? EMBO (Eur. Mol. Biol. Organ.) J. 13:3533-3541.

41. Liu, F., F. Ventura, J. Doody, and J. Massague. 1995. Human type II receptor for bone morphogenic proteins (BMPs): extension of the two-kinase receptor model to the BMPs. Mol. Cell. Biol. 15:3479-3486.

42. Graff, J.M., A. Bansal, and D.A. Melton. 1996. Xenopus Mad proteins transduce distinct subsets of signals for the TGF beta superfamily. Cell. 85:479487.

43. Zhang, Y., X.-H. Feng, R.-Y. Wu, and R. Derynck. 1996. Receptorassociated Mad homologues synergize as effectors of the TGF- $\beta$ response. $\mathrm{Na}$ ture. 383:168-172.

44. Bhushan, A., H.Y. Lin, H.F. Lodish, and C.R. Kintner. 1994. The transforming growth factor beta type II receptor can replace the activin type II receptor in inducing mesoderm. Mol. Cell. Biol. 14:4280-4285.

45. Sanvito, F., P.-L. Herrera, J. Huarte, A. Nichols, R. Montesano, L. Orci, and J.-D. Vassalli. 1994. TGF- $\beta 1$ influences the relative development of the exocrine and endocrine pancreas in vitro. Development. 120:3451-3462.

46. Boettinger, E.P., J.L. Jakubczak, I.S.D. Roberts, M. Mumy, P. Hemmati, K. Bagnall, G. Merlino, and L.M. Wakefield. 1997. Expression of a dominant-negative mutant TGF- $\beta$ type II receptor in transgenic mice reveals essential roles for TGF- $\beta$ in regulation of growth and differentiation in the exocrine pancreas. EMBO (Eur. Mol. Biol. Organ.) J. 16:2621-2633.

47. Shull, M.M., I. Ormsby, A.B. Kier, S. Pawlowski, R.J. Diebold, M. Yin, R. Allen, C. Sidman, G. Proetzel, D. Calvin, et al. 1992. Targeted disruption of the mouse transforming growth factor- $\beta 1$ gene results in multifocal inflammatory disease. Nature. 359:693-699.

48. St-Onge, L., B. Sosa-Peneda, K. Chowdhury, A. Mansouri, and P. Gruss. 1997. Pax6 is required for differentiation of glucagon-producing $\alpha$-cells in mouse pancreas. Nature. 387:406-409.

49. Pituello, F., G. Yamada, and P. Gruss. 1995. Activin A inhibits Pax-6 expression and perturbs cell differentiation in the developing spinal cord in vitro. Proc. Natl. Acad. Sci. USA. 92:6952-6956.

50. Ahlgren, U., S.L. Pfaff, T.M. Jessell, T. Edlund, and H. Edlund. 1997. Independent requirement for ISL-1 in formation of pancreatic mesenchyme and islet cells. Nature. 385:257-260. 